Kraft nähert, immer diejenige ist, welche sich auf den Fall bezieht, dafs die elektromotorische Kraft des Elements constant und von der Intensităt des durchgehenden Stromes unabhängig geworden ist.

VIII. Die Häufigkeit der Farbenveränderungen beim Funkeln der Sterne steht gewöhnlich in Beziehung zur spectralamalytischen Beschaffenheit ihres Lichts;

von Mrn. Ch. Montigny,

Prof am Athensenm zu Brüssel.

(Mitgetheilt vom Hrn. Verf. aus dem Bull. de l'acad. de Belgique SGr. II T. $X X X V I I$.

Die Thatsachen und Ideen, die mich beim Studium der im Titel dieses Aufsatzes genannten Beziehung geleitet haben, kann ich nicht genauer und vollständiger angeben als durch Wiederbolung der folgenden Stelle aus einer meiner fruheren Untersuchungen über das Funkeln.

„In dem Lichte eines Fixsterns fehlen gewisse Strahlen, diejenigen, die den dunklen Linien entsprechen, welche die Spectral-Analyse bei den meisten Sternen entschleiert und deren Anzahl, relative Intensität und Brechbarkeit von einem Stern zum andern verschieden ist. Das Spectrum des Sirius z. B. enthält zwei dunkle Linien im Blau und eine sehr hervorstehende im Grün. Die Abwesenheit der diesen Linien entsprechenden Strablen mufs gewisse Phasen des mit blofsem Auge beobachteten Funkeln dieses schönen Sterns ergreifen. In der That, im Augenblick, wo jeder andere Strahl als die blauen aufgefangen ist, ist demzufolge der Einflufs der blauen Strablen in der complementaren Farbe, welche das Bild des Sirius vorübergehend bekleidet, etwas verschieden von dem was es seyn würde, wenn diese 
Tarbe nicht beraubt wäre der blauen Strablen, die den beiden bezeichneten Linien in dem Theile des Spectrums dieses Sternes entsprechen. Der Einflufs der beim Funkeln eines Sternes fehlenden Strahlen, welcher die eigene Farbe desselben modificiren mufs und von dem ich soeben das einfachste Beispiel citirte, ist bisher noch nicht besprochen worden." ${ }^{\text {I) }}$

Nach dieser Einleitung machte ich in derselben Arbeit bemerklich, dafs die Menge der Linien, welche nach den Messungen der HH. Huggins und Miller, sowie des Paters Secchi, das Roth, Grün und Blau des Spectrums von $\alpha$ Orion charakterisiren, erlaubt, der Abwesenheit einer grofsen Anzahl von Strablen in dem durch unsere Atmosphäre zerstreuten Lichte dieses Sternes die Unregelmäfsigkeiten zuzuschreiben, welche das Funkeln desselben darbietet und in eigenthlimlicher Weise rom Hrn. Prof. Dufour, in Morges, beschrieben sind in der vor einigen Jahren der Akademie gemachten wichtigen Mittheilung taber das Phănomen des Funkelns. ${ }^{2}$ ) In der That, der Einflufs des im Lichte des Sternes $\alpha$ Orion fehlenden Stroms tritt bei dem Funkeln in zweierlei Weise auf: zunächst in der Natur der complementaren Farben, die das funkelnde Bild vorübergehend besitzt, wie eben rom Sirius gesagt ist, und dann in den vielen Lucken, welche aus der Abwesenheit der den Spectrallinien in den Farbenbündeln des Sternes $\alpha$ entsprechenden Strahlen hervorgehen, welche Bündel die Atmosphare durch Dispersion trennt, bevor sie sich im Auge des Beobachters vereinigen. Man begreift nämlich, wie ich auch gesagt habe, dafs vermöge des Einflusses dieser Lücken die Phasen des Funkelns des Sternes $\alpha$ weniger hăufig und weniger regelmăfsig

1) Notice sur la séparation des trajêctoires detrits dans l'atmosphère par les myons de même origine sidérale, mais de refrangibilüé différente, et sur les effets de cette séparation à l'égard de la scintillation (Bull. de l'Acad, royal de Belgique, Ser. II, T. XXIX, 1870.

2) Sur la scintillation des etoiles. Lettre à M. Quetelet par M. Ch. Dufour, Professear à Morges en Suisse (Ib. Ser. I, T. XXIII). 
werden, als sie bei einem anderen an Lichtstrahlen reicheren Stern seyn würden; denn da der Durchgang einer Luftwelle durch eine dieser schmalen Lücken die darin mangelnden Strablen nicht afficiren kann, so kann auch dieser Durchgang die Farbe und den Glanz des Sternbildes in keiner Weise verăndern, entgegen dem, was bei einem an Lichtstrahlen reicheren Gestirn stattfinden würde.

Setzen wir hinzu, dafs diese mehr oder weniger der Lichtstrablen beraubten Lücken in den Farbenbündeln eines Sterns, dessen Spectralbild viele und starke Linien zeigt, sich in unserer Atmosphäre in dem Maafse verbreitern, als der Stern sich dem Horizonte nähert und zwar je nach der Brechbarkeit der Strahlen, welche jede der Läcken begränzen. Hienach ist es nicht überraschend, dafs die Curve, welche das Funkeln von a Orion bei verschiedenen Höben vorstellt, Hrn. Dufour bedeutendere Unregelmäfsigkeiten zeigten als die, welche bei seinen Untersucbungen die Funkelcurven anderer Sterne darboten, z. B. Capella, eines Sterns mit feinen und wenig zahlreichen Spectrallinien, dessen Funkeln von diesem Physiker als das regelmälsigste angegeben wird.

Die Beziehungen, die ich somit anfangs aufstellte zwischen den Charakteren des Funkelns der beiden angeführten Sterne und der Beschaffenheit ihres respectiven Lichts gemäls der Spectralanalyse, haben mich zu einem besonderen Studium dieser Aufgabe veranlafst. Ich habe, so weit es der Zustand des Himmels und meine übrigen Beschäftigungen es erlaubten, nahe bei Brüssel das Funkeln von 41 Sternen studirt, von denen die Zusammensetzung des Lichts durch Spectralanalyse ziemlich wohl bekannt ist. Die Resultate meiner bis in die jüngste Zeit fortgesetzten Beobachtungen sind durchaus den anfänglich aufgestellten Beziehungen günstig und daher beehre ich mich hier sie in Kurze der Akademie mitzutheilen.

Zuvörderst mufs ich jedoch sagen, dafs darin die Thatsachen mit absoluter Unabhängigkeit von jeder Theorie des Funkelns behandelt seyn werden. Ich werde also nicht 
untersuchen, ob diese charakteristischen Verănderungen in Farbe und Glanz beim Funkeln der Sterne durch Interferenzphänome entstehen, wie nach A ra g o's Theorie, oder ob die Sternstrablen, nach meiner Erklärung, durch eine totale Reflexion an der Oberfläche der Luftwellen plötzlich aufgefangen werden, oder ob, wie es Andere wollen, diese Veränderungen einfach aus Ungleichheiten in Refraction und Dispersion entspringen, welche die Strablen eines Sterns dadurch erleiden, dafs diese beweglichen Luftwellen in ihre Bahn eintreten. Ich habe nicht die Absicht mich mit dieser theoretischen Frage zu beschäftigen, werde vielmehr in einer allgemeinen Arbeit, welche die Gesammtheit aller meiner Beobachtungen über das Funkeln umfafst, darauf zurủckkommen. In dem, was folgt, stütze ich mich hauptsächlich auf zwei unbestreitbare Thatsachen: 1) dafs die verschiedenen Farbenstrablen eines selben Strables durch atmosphärische Dispersion getrennt werden, ehe sie in das Auge oder das Fernrohr des Beobachters eindringen; 2) dafs zwischen diesen Strablen Lücken vorhanden sind, welche je nach Breite, Intensităt und Anzahl der das Spectrum des betrachteten Sterns durchziehenden Linien, mehr oder weniger hervortreten.

Meine Beobachtungen umfassen zwei geschiedene Perioden; die eine, 47 Abende begreifend, erstreckt sich vom October 1870 bis Ende des März 1871, und die andere, 19 Abende bildend, hat zu Gränzen den Ausgang Junis und den Anfang Decembers $1873^{1}$ ). In beiden Reihen

1) Verschiedene Verhinderungen haben diese beiden Beobachtungsreihen getrennt, was ich um so mehr bedaure, als die am Schlufs der ersteren erhaltenen Resultate schon klar bewiesen, dafs das Funkeln derjenigen Sterne, die am meisten durch die Charactere der Linien in ihren Spectralbildern von einander abweichen, verschieden ist. Die damalige Jahreszeit zeigte sich so ungünstig für diese Untersuchungen, dals ich mich entschlofs, die zweite Periode momentan abzubrechen und zunächst die vereinigten Resultate beider Reihen zu veröffentlichen, die, wie man weiterhin sehen wird, mit denen der ersten vollkommen übereinstimmen. 
begannen die Beobachtungen mit den Abendstunden und erstreckten sich bis 11 Uhr Nachts. Die Beobachtungen an Ende December 1870 und Anfang des folgenden Monats waren durch sebr niedrige Temperaturen charakterisirt, die beim Ende der Beobachtungen am 1. und 4. Januar $-9^{\circ}$ und $-10^{\circ}$ erreichten.

Das Instrument, dessen ich mich bediente, ist ein astronomisches Fernrohr, das eine Vergröfserung von 85 gewährt und ein Objectiv von $77 \mathrm{Mllm}$. Durchmesser hat. Zwischen dem Objectiv und dem Ocular, letzterem sehr nahe, ist das Scintillometer angebracht, welches ich ersann nnd fruher beschrieben habe ${ }^{1}$ ). Ich begnüge mich hier mit der Angabe, dafs das Instrument wesentlich aus einer dicken kreisrunden Glasplatte besteht, schief vor den Linsen des Oculars befestigt an einer Rotationsaxe, die der Axe der Figur des Fernrohrs parallel ist. Diese Platte durchschneidet das gegen das Ocular convergirende Lichtbündel bei allen Lagen, welche es rings um seine Rotationsaxe annimmt. Letztere wird in Bewegung gesetzt durch einen Mechanismus, der aufserhalb des Körpers des Fernrohrs angebracht ist und durch einen $Z$ ügel (frein) regulirt wird. Ein sich mit ihm bewegender Zeiger erlaubt, die Zahl der Umdrehungen, welche die Platte in gegebener Zeit vollführt, zu berechnen. Man sieht leicht ein, dafs die continuirliche Dazwischensetzung der Platte unter Neigungen, die in Bezug auf denselben Strahl während eines vollen Umlaufs und zwar dicht am Scheitel der Convergenz der Strahlen des Sterns regelmäfsig variiren, das Bild desselben einen vollen Kreis im Fernrohr beschreiben lassen mufs. Wenn der Stern nicht funkelt, hildet er einen continuirlichen Strich, der die Farbe des Sterns besitzt; wenn er aber funkelt, zerfällt diese Curve in lebhaft gefärbte Bögen, die sich rasch verändern, und in welchen gewöbnlich Roth, Gelb, Grūn und Blau, zuweilen Violett erglänzen, je nach den Charakteren des Funkelns, die abbängen von der $\mathrm{Na-}$

1) Bull. de l'Acad. royal de Belgique, Serr. $I, T$, XVII. 
tur des Sterns, seiner Höhe und den atmosphărischen Umstānden wăhrend der Beobachtung ${ }^{1}$ ).

Um die so wechselnden und so flüchtigen Farbenbögen möglichst genau zu zählen, bringe ich im Brennpunkt des Oculars des Fernrohrs ein Mikrometer an, bestehend aus drei feinen Fäden, die sich diametral in der Weise schneiden, dafs sie in dem kreisrunden Feld des Instruments secbs gleiche, paarweise gegenüberstehende Sectoren bilden, von denen jeder ein Sechstel des kreisrunden Feldes einnimmt. Nachdem dies Mikrometer bei jeder Beobachtung zweckmäfsig beleuchtet ist, fübre ich sein Centrum oder den Kreuzpunkt der Fäden auf einen Punkt des vom Bilde des funkelnden Sterns beschriebenen Unkreises. Die Hälfte der Anzahl von Färbungen, welche in einem gegebenen Moment auf dem Bogen zwischen den die Seiten eines der Sectoren bildenden Fäden erscheinen, zeigt offenbar die Anzahl der Veränderungen an, die das Sechstel des Umkreises zerfällen, welcher ähnliche Farbenbögen in seiner ganzen Erstreckung darbietet. Diese Angabe, und die zu einer Umdrehung der geneigten Platte erforderliche Zeit, welche ich mittelst eines mit Hemmung versehenen Secunden-Chronometers unmittelbar aus der während 39 Umläufen verflossenen Zeit ableitete, erlaubten mir, genau die Anzahl der Farbenveränderungen zu berecbnen, welche das Bild des funkelnden Sterns während einer Secunde erleidet.

Bei diesen Beobachtungen notire ich gleichfalls die verschiedenen Farben, welche die Circumferenz zerfällen, sey es, dafs es einige giebt, die den sebr kurzen Momenten entsprechen, wo der Stern seine natürliche Farbe annimmt, sey es, dafs die meisten wesentlich von dieser abweichen,

1) Die kreisrunde Glasplatte des Scintillometers, die ich gröfstentheils selbst verfertigte, hat einen Durchmesser von $47^{\mathrm{mm}}$ und eine Dicke von $6^{\mathrm{mm}}, 4$. Sie neigt etwa $13 \frac{1}{2}^{\circ}$ gegen die Rotationsaxe. Der im Fernrohr von dem Sternbilde beschriebene Kreis hat bei gewöhnlicher deutlicher Gesichtsweite einen Durchmesser von $23^{\mathrm{mm}}$, wie ich berechnete und durch den Versuch bestätigte. 
was am häufigsten geschieht. Alle diese successiven Farben bilden die Farbenveränderungen, von denen in gegenwărtiger Arbeit die Rede ist.

Was die Zenitbdistanz des Sterns im Moment der Beobachtung betrifft, ein zu kennen wichtiges Element, wie wir sehen werden, so bestimme ich es mittelst eines besonderen Instruments, ehe ich die farbigen Bögen beobachte.

Bekanntlich ist das Funkeln eines Sterns desto lebhafter als, unter sonst gleichen Umständen, die Zenithdistanz desselben grölser ist. Die Beobachtungen, die in einem Abend an verschiedenen Sternen bei sehr verschiedenen Zenithdistanzen angestellt werden, würden also unter sich nicht vergleichbar seyn, wenn wir nicht mittelst eines gewissen Calcüls die Anzahl der Farbenveränderungen, welche das Funkeln eines Sternes charakterisiren, reduciren könnten auf Werthe, die einer ausgewählten und für alle Sterne gleichen $Z$ enithdistanz entsprechen. Der Reductionscalcũl geschieht leicht mittelst des zweiten der Gesetze, welche Hr. Dufour aus seinen vielen Untersuchungen über das mit blofsem Auge beobachtete Funkeln abgeleitet hat, wenn man dabei den von diesem Physiker eingeschlagenen Weg verfolgt'). Er hat bewiesen, dafs, aufser dicht beim Horizont, das Funkeln nahezu proportional ist dem Product aus der Dicke der vom Strahl des Sterns durchlaufenen Luftschicht in die astronomische Brechung, welche der beobachteten Höhe entspricht. Be-

1) Ich halte es für nützlich, die drei Gesetze, welche Hr. Da four, zu Morges, aus einer Reihe von 13000 mit blofrem Auge gemachten Beobachtungen abgeleitet hat, hier anzuführen.

1) Unter sonst gleichen Umständen funkeln die rothen Sterne weniger als die weifsen.

2) Aufser dicht beim Horizont, ist das Funkeln proportional dem Product aus der Dicke der Luftschicht, welche der Lichtstrahl des Sterns durchwandert hat, in die zugehörige astronomische Refraction der betrachteten Höhe.

3) Aufser dem Einflufs der Farben giebt es bei dem Funkeln der Sterne noch andere wesentliche Untersohiede, die von den Sternen gelbst herturühren soheinen. 
zeichnet man demnach mit $Q$ und $Q^{\prime}$ die Producte, die zweien gegebenen Zenithdistanzen entsprechen, für welche die Intensităten des Funkelns eines Sterns an einem selben Abend respective $S$ und $S^{\prime}$ sind, so hat man

$$
S=S^{\prime} \frac{Q}{Q^{\prime}}
$$

Ist $Q^{\prime}$ ausgedrückt in Theilen des zur Einheit angenommenen Products $Q$, welches der Zenithdistanz entspricht, auf die man durch Rechnung die Intensität $S$ des Funkelns zurückführen will, so hat man $S$ ausgedrückt in Function von $S^{\prime}$, welche die Intensität des Funkelns ist, welche wirklich bei der dem Producte $Q^{\prime}$ entsprechenden Zenithdistanz beobachtet worden ist, mittelst der Formel:

$$
S=\frac{S^{\prime}}{Q^{\prime}} .
$$

Hr. Dufour hat in den Annales de la Société vaudoise des sciences naturelles (Année 1860 No. 47) ${ }^{1}$ ) eine Reductionstafel gegeben, berechnet für zehn zu zehn Grad Höhe des Sterns und in der Annahme einer Höhe der Atmosphäre von $\frac{1}{80}$ Erdradius oder ungefähr 79,5 Kilometern. Ich habe eine ähnliche Tafel construirt, berechnet für jeden Grad Zenithdistanz. Sie hat mir dazu gedient, die Anzahl der Farbenveränderungen, welche ein Strahl bei verschiedenen Distanzen an einem selben Abend erleidet, zuräckzuführen auf diejenige Anzahl von Veränderungen, die er bei $60^{\circ}$ Zenithdistanz erleiden würde ${ }^{2}$ ).

1) Instruction pour lobservation de la scintillation des étoiles, par M. Chärles Dufour.

2) Es ist hier der Ort zu bemerken, dafs Brn. Du fou r's Art, die Intensität des Funkelns zu charakterisiren, auf den ersten Blick von der aus meinem Verfahren hervorgehenden abweicht. Um diesen wichtigen Punkt zu erläutern, will ich hier die Anleitung hersetzen, die Hr. Dufour in Betreff des seinigen gegeben hat. "Es ist leicht", sagt er, "sobald man etwas daran gewöhnt ist, ziemlich genau zu schätzen, ob ein Stein mehr oder weniger als ein anderer funkele und man kann dieses Funkeln durch eine $Z$ iffer ausdrücken, wie man in der Meteorologie den Zustand des Himmels oder die Stärke des Windes durch eine Ziffer angiebt. Meinerseits bezeichne ich mit 0 
Als ich mein Studium des Funkelns in seinen Beziehungen zur Zusammensetzung des spectralen Lichts der Sterne anfing, ging meine erste Sorge dahin, mich zu versichern, ob das zweite Gesetz des Hrn. Dufour eben so gut für die mit dem Scintillometer gemachten Beobachtungen gelte wie für die mit blofsem Auge vollzogenen Messungen, und ob die Anzahl der für einen selben Stern, an

das Funkeln Null und mit 10 das Funkeln, welches mir am stärksten erscheint. Solche Scintillationen kommen selten vor, nur wenn die Sterne dicht am Horizont stehen, scheinen sie zu hüpfen, die Farbe zu wechseln und bisweilen $z u$ verschwinden. Mit etwas Uebung gelingt es noch Grade des Funkelns zwischen 0 und 1, 1 und 2 zu unterscheiden."

Der Hauptcharakter, der, für mich, die Stärke des Funkelns bei Untersuchungen mit dem Scintillometer milst, ist die Anzahl der Farbenveränderungen des Sternbildes innerhalb einer Secunde, geschätzt nach der Menge von Farbenbögen, in welche der von diesem Bilde im Fernrohr beschriebene Kreis zerfällt. Wenn die Dauer des Umlaufs der geneigten Glasplatte nicht $0^{\prime}, 34$ übersteigt, was, nach Hrn. Platean, durchschnittlich die Gesammtdauer eines Eindrucks auf die Netzhaut ist, so beschreibt das Bild des Sterns einen geschlossenen Kreis; alsdann entspringt jede Farbe, die man während eines Umlaufs der Scheibe wahrnimmt, ans einem einzigen Licht-Eindruck, und dieser bleibt fast ganz unabhängig von dem Eindruck, welcher während des vorhergehenden Umlanfs an demselben Ort der Netzhaut erregt worden ist, wenn die Umlaufsgeschwindigkeit der Scheibe eine gewisse Gränze nicht überschreitet.

Bei den Beobachtungen mit blofsem Auge ist es unmöglich, dafs dieses Organ die einzelnen Licht-Eindrïcke unterscheide, wenn diese einander mit der Schnelligkeit überdecken, welche die Farbenveränderung des Bildes eines funkelnden Sternes characterisirt. Im Allgemeinen kann das Ange auch nur die hervorstechendsten Effecte dieser Ueberdeckung der verschiedenen Eindrücke wahrnehmen.

Ungeachtet der Verschiedenheit, welche hiernach zwischen den beiden Beobachtungsweisen des Funkelns stattfindet, bin ich doch geneigt zu glauben, dafs sie durch innige Proportionalitätsbeziehungen mit einander verknüpft sind. Wir werden nämlich sehen, dafs die relativen Intensitäten des Funkelns von sechs Hauptsternen, die $\mathrm{Hr}$. Dufour mit blossem Ange geschätzt hat, genau dieselbe Ordnung befolgen wie die an denselben Sternen mit dem Scintillometer gemessenen absoluten Intensitäten. Bei Prokyon und Vega sind bei jeder die Bestimmungen genan gleich. 
einem selben Abend, bei verschiedenen Zenithdistanzen erhaltenen Veränderungen, für die Distanz $60^{\circ}$, welche icb zur Reduction meiner Beobachtungen gewählt, auf denselben Werth zurückkommen würden. Die folgende Tafel (S. 287) enthält Beispiele von dieser Rechnung, angewandt auf das Funkeln von sechs Hauptsternen.

Die durch Rechnung auf $60^{\circ}$ Zenithdistanz reducirten Veränderungen stimmen für einen selben Stern und einen selben $A$ bend meistens sehr genügend mit einander, obgleich die bei verschiedenen Zenithdistanzen gemessenen absoluten Zahlen oft vom Einfachen aufs Doppelte und Dreifache stiegen (Capella, am 17. October). Die scheinbaren Zenithdistanzen schwankten freilich kaum von $8^{\circ}$ auf höchstens 10"; dies rührt daher, dafs meine Beobachtungen gewöhnlich $z$ wischen $50^{\circ}$ und $72^{\circ}$ angestellt wurden. Bei geringeren Zenithdistanzen als etwa $50^{\circ}$, sind in meinem Fernrohr von $77 \mathrm{Mllm}$. Durchmesser die Farben nicht mehr recht deutlich, aufser bei sebr grofser Kälte. Dieser Mangel an Deutlichkeit entspringt aus dem Einflufs, den der Durchmesser des angewandten Fernrohrs auf die Anzahl und den Glanz der Farbenveränderungen bei dem Funkeln ausübt, wie ich durch Theorie und Erfahrung gezeigt habe. ${ }^{1}$ ) Andrerseits entsprechen die reducirten Werthe, unterhalb $72^{\circ}$ bis $74^{\circ}$, nicht mehr einander, wie schon Hr. Dufour bemerkt hat.

Die vorstehenden Entwickelungen waren durchaus un-

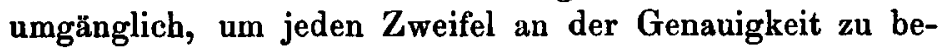

1) Bull. de l'acad. royal de Belgique, Ser. II, T. XXIX, p. 80 et 455. Ich betrachte hier nicht im Detail die Häufigkeit dieser oder jener Farbe in dem zu einem Kreis ausgebreiteten Bilde eines funkelnden Sternes. Die Farben wechseln nicht allein pach dem eignen Farbenton des Sterns, sondern auch nach seiner Höhe über dem Horizont und nach dem so vorwaltenden Einflufs der atmosphärischen Zustände. In dieser Beziehung bemerke man in obiger Tabelle, wie die Häufigkeit des Funkelns bei einem selben Stern, z. B. bei Prokyon, von einem Abend zum andern geschwankt hat. Ich behalte mir vor, diese Eigenthümlichkeiten in anderen Arbeiten über das Funkeln zu beschreiben. 
287

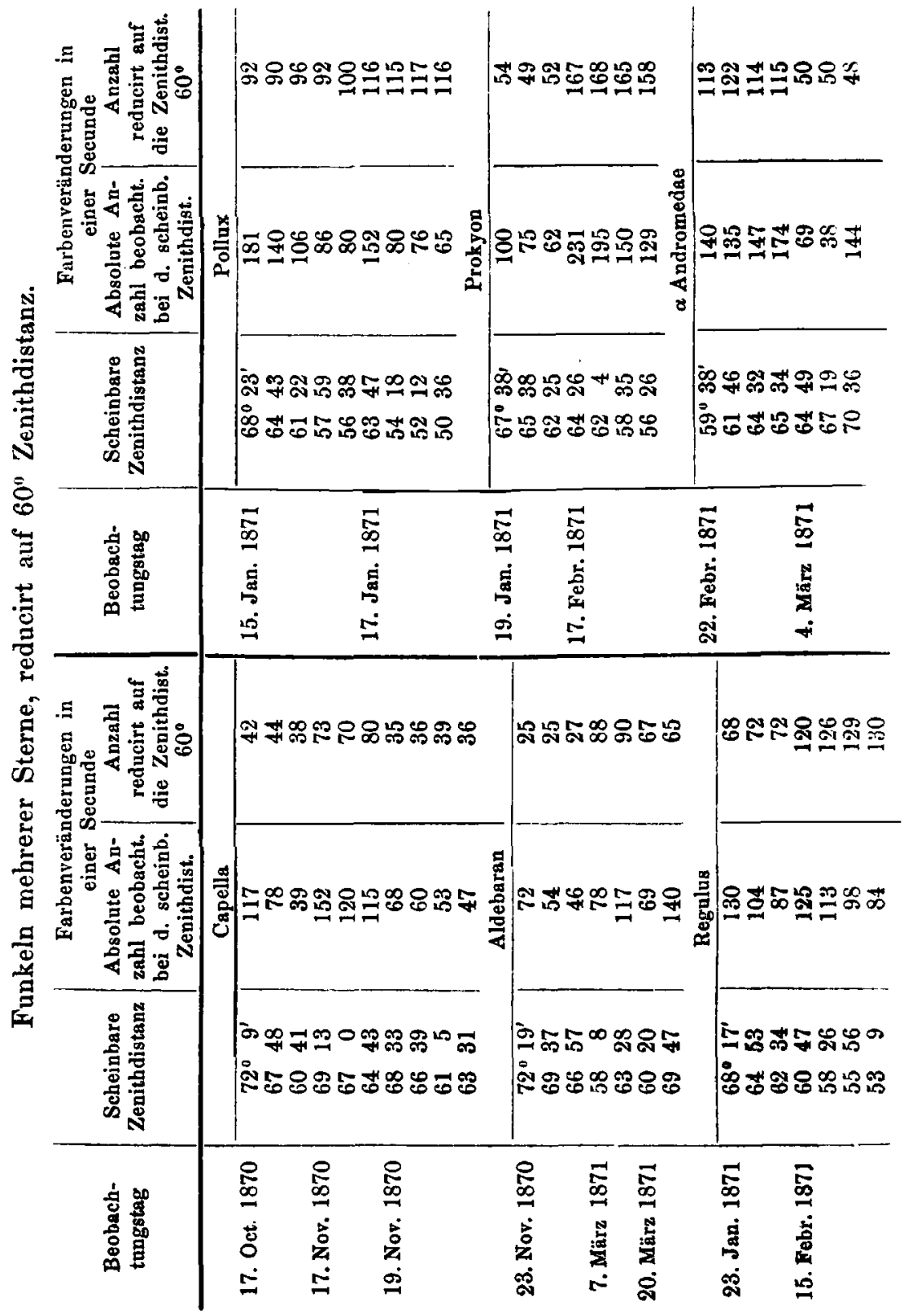


seitigen, welche die Messung der Hăufigkeit des Funkelns in der Frage über ihre Beziehungen zur spectralen Zusammensetzung des Lichts der Sterne beansprucht.

Ich werde bei dieser Vergleichung der Classification folgen, nach welcher Pater Secchi für 234 Sterne, gestützt auf die Anzahl und den Charakter der Linien in ihren spectralen Bildern, drei Haupttypen annimmt. Von diesen Sternen habe ich 41 ausgewählt, die Gegenstand meiner Messungen des Funkelns waren. Die unterscheidenden Kennzeichen der Typen, auf welche Hr. Secchi die beobachteten Sternspectren bezieht, hat derselbe der Pariser Academie im September 1866 mitgetheilt.') Aus dem Werke „Soleil“, welches der italienische Astronom neuerlich veröffentlicht hat, will ich die Hauptangaben über diese Typen entlehnen.

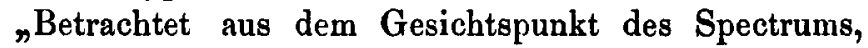
welches sie liefern, beziehen sich die Sterne auf vier vollkommen gesonderte Typen; einige wenige Spectren, die sich nicht scharf in diese Kategorie bringen lassen, scheinen Uebergänge $z$ wischen ihnen zu bilden.

„Der erste Typus ist der der weilsen Sterne, wie Sirius, Vega, Ataï, Regulus, Rigel, die des grol'sen Bären mit Ausnahme vom $\alpha$, die des Schlangenträgers u. s. w. Alle diese Sterne, welche man weifse nennt, obwohl sie in Wirklichkeit schwachblau sind, geben ein Spectrum, gebildet aus den gewöhnlichen sieben Farben, und unterbrochen durch vier starke schwarze Linien, eine im Roth, eine im Grünblau, und zwei im Violett. Diese vier Linien gehören dem Wasserstoff an. Aufser diesen fundamentalen und sehr breiten Linien sieht man bei den hellsten Sternen, z. B. beim Sirius, eine schwarze sehr feine Linie im Gelb, die mit der des Natriums zusammen zu fallen scheint, und in dem Grün schwächere Linien, die dem Magnesium und dem Eisen angehören. Die auffallendste Eigenthümlichkeit dieses Typus ist die Breite gewisser Linien. Fast die Hälfte aller Sterne des Himmels gehört diesem Typus an."

„Der zweite Typus ist der der gelben Sterne, wie Ca1) Ann. Bd. 131, S. $156 . \quad$ P. 
pella, Pollux, Arcturus, Aldebaran, $\alpha$ Ursae majoris, Prokyon u. s. w. Das Spectrum dieser Sterne ist vollkommen dem unserer Sonne ähnlich, d. h. es ist gebildet aus sehr feinen und sehr dicht liegenden Linien, welche dieselbe Lage haben wie im Sonnenspectrum. Im Spectrum des Pollux und der Capella sind diese schwarzen Linien äufserst fein; breiter und leichter erkennbar sind sie beim Arctur und Aldebaran. Dieser letzte Stern könnte sogar als Uebergang vom zweiten zum dritten Typus betrachtet werden, während Prokyon zwischen dem ersten und zweiten stände. Wir haben gesagt, dals der erste Typus fast die Hälfte aller bisher beobachteten Sterne enthalte; zwei Drittel der übrigen müssen in die Kategorie der eben besprochenen gelben Sterne gestellt werden.

„Das Spectrum des dritten Typus ist etwas ungewöhnlich; es besteht aus einem doppelten Systeme von nebligen Streifen und schwarzen Linien. Als Beispiel desselben kann man $\alpha$ Herculis nehmen. Wirklich sind die schwarzen fundamentalen Linien dieselben wie im zweiten Typus, was man besonders am Arcturus und Aldebaran erkennt, aber aufserdem enthält der dritte Typus eine grofse Anzahl nebliger Streifen, welche dieses Spectrum zerschneiden und eine Art von Colonnade bilden. Diese Streifen, deren Breite und Intensität sehr verschieden sind, bilden bei den Sternen dieser Kategorie ziemlich bedeutende Unterschiede. Als fundamentalen Typus haben wir $\alpha$ Herculis gewählt, weil dieser Stern das regelmäl'sigste Spectrum bildet. Wir können noch erwähnen $\beta$ im Pegasus, $\varepsilon$ im Wallfisch, $\alpha$ im Orion, Antares usw. Diese Sterne sind sehr merkwürdig, denn sie sind ganz veränderlich und besitzen eine mehr oder weniger ins Rothe oder Orange fallende Farbe. Bei einigen kleineren Sternen sieht man, statt der Colonnaden, Gruppen von bellen Linien, getrennt durch dunkle Räume... Die schönen Sterne dieses Typus sind nicht sehr zahlreich; der merkwürdigsten giebt es etwa dreifsig und wenn Inan die des zweiten Typus dazu rechnet, haben wir höchstens ein Hundert gefunden.

Poggendorff's Annal, Bd. CLIII. 
Das sind die hauptsächlichsten Kennzeichen der drei ersten Typen des Pater Secchi. Er nimmt noch einen vierten an, welcher die kleinen ziemlich zablreichen Sterne von blutrother Farbe umfafst. Keiner derselben war Gegenstand meiner Beobachtungen. Unter den 41 Sternen, auf die ich meine Aufmerksamkeit richtete, sind wenige unterhalb der dritten Grölse. Die Sterne von schwachem Glanz zeigen die Farben nicht so deutlich, dafs ich sie mit meinem Gesicht sicher zählen konnte.

Die nebenstehende Tafel enthält die von mir studirten Sterne, klassificirt nach den drei Typen des Pater Secchi und geordnet, bei jedem, nach der mittleren Anzahl der Farbenveränderungen, welche sie bei den Messungen während der vereinigten Dauer meiner beiden Beobachtungsperioden darboten. Diese Zahlen sind durch Rechnung auf die Zenithdistanz $60^{\circ}$ reducirt. Diesen Resultaten habe ich hinzugefügt erstlich, die gewöhnlichen Gröfsen der Sterne nach Sir John Hersehel, dann die Anzahl der Abende, an denen jeder Stern beobachtet wurde, und endlich die gesammte Anzahl von Beobachtungen, deren Gegenstand er war. ${ }^{1}$ )

1) Es ist wichtig hier zu sagen, dafs der Typus der gelben Sterne mit feinen Linien oder schwachen Streifen, der in der Mittheilung des Pater Secehi an die Pariser Akademie im September 1866 der dritte war, in dem Werke: Le Soleil despelben Gelehrten der zweite geworden ist. Diese letzte Ordnung ist es, die ich angenommen habe.

In der folgenden Tafel umfarst jeder Typus die Sterne, die sich nach der eben erwähnten Mitheilung darauf beziehen, bis auf die folgenden Ausnahmen.

1) Arcturus, welcher anfangs im zweiten Typus, dem der Sterpe mit feinen Streifen, stand, ist jetzt vom Pater $\mathrm{Secchi}$ in dem Werke Le Soleil in den dritten, oder den mit schwarzen Streifen versetzt.

2) Prokyon steht nach Hrn. Secchi zwischen dem ersten und zweiten Typus, Aldebaran dagegen zwischen dem zweiten und dritten. Ich habe Prokyon in den ersten Typus gestellt und Aldebaran in dep dritten, wo er zusammentrifft mit Arcturus, der, nach Hrn. Secchi, dieselben Eigenthümlichkeiten in seinem Spectrum darbietet.

Am Schlusse des zweiten Typus habe ich die drei Sterne des Orions rereinigt, weil bis auf Betelgeuze, die Sterne dieses Sternbildes eine 
Sterne, klassificirt nach der Beschaffenheit ihres Lichtes und der Hăufigkeit ihres Funkelns.

\begin{tabular}{|c|c|c|c|c|c|c|c|c|c|c|c|c|c|c|}
\hline \multicolumn{5}{|c|}{$\begin{array}{l}\text { Erster Typus, } \\
\text { Sterne mit vier Linien im Spectrum. }\end{array}$} & \multicolumn{5}{|c|}{$\begin{array}{l}\text { Zweiter Typus, } \\
\text { inen Linien und schwachen Streifen. }\end{array}$} & \multicolumn{5}{|c|}{$\begin{array}{l}\text { Dritter Typus, } \\
\text { Streifen und }\end{array}$} \\
\hline \multirow[b]{2}{*}{ Sterne } & \multirow{2}{*}{ 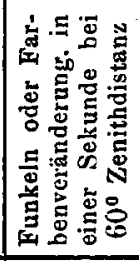 } & \multirow{2}{*}{ 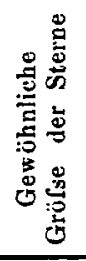 } & \multicolumn{2}{|c|}{ Zahl } & \multirow[b]{2}{*}{ Sterne } & \multirow{2}{*}{ 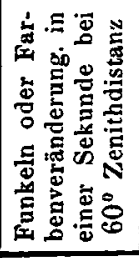 } & \multirow{2}{*}{ 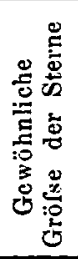 } & \multicolumn{2}{|c|}{ Zahl } & \multirow[b]{2}{*}{ Sterne } & \multirow{2}{*}{ 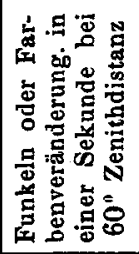 } & \multirow{2}{*}{ 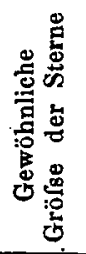 } & \multicolumn{2}{|c|}{ Zahl } \\
\hline & & & $\begin{array}{l}\text { der Be- } \\
\text { obach- } \\
\text { tungs- } \\
\text { abende }\end{array}$ & $\begin{array}{c}\text { der } \\
\text { Beob- } \\
\text { achtung. }\end{array}$ & & & & $\begin{array}{l}\text { der Be- } \\
\text { obach- } \\
\text { tungs- } \\
\text { abende }\end{array}$ & $\begin{array}{c}\text { der } \\
\text { Beob. } \\
\text { achtung. }\end{array}$ & & & & $\begin{array}{c}\text { der } \mathrm{Be}- \\
\text { obach- } \\
\text { tungs- } \\
\text { abende }\end{array}$ & $\begin{array}{c}\text { der } \\
\text { Beob- } \\
\text { achtung }\end{array}$ \\
\hline ह) im grofsen & 111 & 1,95 & 4 & 4 & $\boldsymbol{\alpha}$ Persens & 98 & 2,07 & 13 & 16 & œPegasus (veränderl.) & 70 & 4 & 4 & 4 \\
\hline${ }_{\beta} \hat{\text { Bären }}$ & 104 & 2,77 & 4 & 5 & Pollux & 96 & 1,60 & 7 & 22 & $\beta$ Pegasus (veränderl.) & 66 & 2,65 & 10 & 15 \\
\hline Prokyon & 103 & 1,00 & 9 & 24 & $\gamma$ Andromedae & 93 & 2,50 & 12 & 12 & Betelgeuze (veründerl.) & 64 & 1,00 & 16 & 32 \\
\hline Vega & 98 & 1,00 & 3 & 10 & Deneb & 89 & 1,90 & 9 & 15 & Aldebaran & 59 & 1,10 & 9 & 38 \\
\hline a Andromedae & 92 & 2,54 & 20 & 32 & Capella & 77 & 2,40 & 17 & 22 & Arcturus & 59 & 0,77 & 15 & 19 \\
\hline Ђ) im groisen & 88 & 2,43 & 10 & 14 & a Widder & 76 & 2,28 & 11 & 19 & $\beta$ Andromedae & 57 & 2,45 & 18 & 29 \\
\hline y Bären. & 87 & 2,71 & 5 & 7 & $\beta$ Stier & 67 & 1,00 & 17 & 35 & $\approx$ Wulfisch (veränderl.) & 56 & $\mathbf{3}$ & 7 & 7 \\
\hline Regulus & 85 & 1,60 & 13 & 24 & $\beta$ Hercules & 65 & 3,18 & 4 & $\tilde{5}$ & Antares & 55 & 1,60 & 3 & 3 \\
\hline Atair & 82 & 1,28 & 8 & 15 & $\beta$ Löwen & 55 & 2,63 & 12 & 17 & $a$ Hercules (veränderl.) & 49 & 3,20 & 4 & 5 \\
\hline a Ophiuchi & 81 & 2,63 & 4 & 5 & $\beta$ Ophiuchi & 42 & 3 & 2 & 2 & E Pegasus & 49 & 2,62 & 2 & 2 \\
\hline Algol & 78 & 2,62 & 15 & 21 & $\alpha$ Wassermann & 40 & 2,97 & 3 & 3 & « Hydra (veränderl.) & 40 & 2,30 & 4 & 5 \\
\hline a). & 73 & 2,65 & 10 & 14 & $\gamma$ Orion & 60 & 2,18 & 15 & 24 & & & & & \\
\hline$y$ (1il r tgasus & 72 & 3,11 & 11 & 12 & $\beta$ Orion, Rigel & 57 & 0,82 & 13 & 21 & & & & & \\
\hline Castor & 62 & 1,94 & 5 & 9 & $\psi$ Orion & 53 & 2,59 & 10 & 14 & & & & & \\
\hline a Coronae & 61 & 2,69 & 12 & 7 & & & & & & & & & & \\
\hline$\eta g$ gr. Bäron & 61 & 2,18 & 8 & 12 & & & & & & & & & & \\
\hline Mittel u. Summo & 86 & 2,07 & & 225 & Mittel u. Summe & 69 & 2,22 & & 227 & Mittel und Summe & 56 & 2,25 & & 159 \\
\hline
\end{tabular}


Aus dieser Tafel ergiebt sich nun hauptsächlich folgendes:

1) Die Sterne, welche am meisten funkeln, gehören zum ersten Typus oder zu demjenigen mit wenig Spectrallinien, während die schwach funkelnden Sterne im Allgemeinen in den dritten Typus oder den mit breiten Spectralzonen fallen.

2) Das Mittel 86 des ersten Typus ubertrifft um vieles das von 56 des dritten. Was das Mittel 69 der zweiten Gruppe betrifft, so liegt es zwischen denen der beiden anderen, doch dem der dritten etwas näher.

3) Obgleich einige Sterne von wenig verschiedener Grölse einander aucb durch die numerischen Intensitäten ihres Funkelns nahe stehen, besonders im ersten Typus, so zeigt sich doch kein merkenswerther Zusammenhang zwischen der Häufigkeit des ersten Phänomens und der Gröfsenordnung der Sterne. Wir sehen sogar die beiden letzten Typen gleiche Mittelgrölsen darbieten, während ihr Funkeln beträchtlich versohieden ist.

Die beiden ersten Folgerungen setzen es sogleich aufser

Unterabtheilung dieses Typus bilden, in Bezug auf welche Pater Secc h i sich in dem genannten Werke (p. 398) folgendermafsen ausdrïckt:

nAufser den vier Haupttypen giebt es Sterngruppen, welche eine besondere Aufmerksamkeit verdienen. Eine solche ist das Sternbild des Orion; es gehört wegen der äulsersten Feinheit seiner Linien zum zweiten Typus, aber zugleich ist es sehr merkwürdig durch die fast rollständige Abwesenheit des Roth und des Gelb, so dafs die Sterne dieser Region einen doppelten Charakter zeigen: 1 . haben sie eine sehr vorwaltende grüne Farbe und 2. sind die Linien in ihrem Spectrum so fein, dafs sie oft schwer $z u$ trennen sind."

Sirius, der schönste Stern des Himmels, so merkwürdig durch den Glanz und die Lebhaftigkeit seines Funkelns für das blofse Auge, steht nicht in der Tafel, weil meine Beobachtung in Betreff desselben relativ wenig zahlreich sind und meistens bei ziemlich hoher Lufttemperatur stattfanden; dieser letzte Umstand mufste den Werth seines Eunkelns, das übrigens ziemlich nahe beim Horizont beobachtet warde, merklich afficiren. Auch $\mathrm{Hr}$. Dufour hat die Intensităt des Phänomens beim Sirius nicht festgestellt, unter andern wegen Nähe des Horizonts. 
Zweifel, dafs die von mir vorausgesetzte Thatsache des Zusammenhanges der Häufigkeit des Funkelns der Sterne mit der Beschaffenheit ihres spectralen Lichts wirklich existirt. Die Sterne, deren Spectrum ein doppeltes System von dunklen Streifen und schwarzen Linien darbietet, und denen folglich die meisten und hervorragendsten Lücken zwischen den durch Dispersion in unserer Atmosphäre getrennten Strahlen entsprechen, funkeln weniger als die Strahlen mit feinen Spectrallinien, und viel weniger als die, welche ein Spectrum mit blofs vier schwarzen Linien zeigen und demnach nur eine sehr kleine Zahl von Lücken $z$ wischen den durch die Luft zerstreuten Lichtbündeln darbieten.

Das mittlere Funkeln der drei Typen steht im Verhältnils der Zablen 14, 11 und 9.

Mit Recht darf man fragen, ob nicht die Temperatur der Luft, welche einen so hervorstechenden Einflufs auf das Funkeln hat, da dieses gemeiniglich um so lebhafter ist, je gröfser die Kälte, zu gewissem Theile mit im Spiele gewesen sey bei den Werthen, welche das Funkeln der drei Sterntypen auf eine so entschiedene Weise charakterisiren. Zufällig könnten die Beobachtungen, welche sich auf den ersten Typus beziehen, bei einer im Allgemeinen niedrigeren Lufttemperatur gemacht worden seyn als die des dritten Typus. Wenn dem so wäre, würde es nicht überraschend seyn, dafs der Mittelwerth für letztere weniger hoch war als der für den ersten Typus? Um auf diesen Einwurf zu antworten, mufs ich zuvörderst bemerken, dafs Sterne wie $a$ und $\beta$ Andromedae, welche respective zum ersten und dritten Typus gehören, ziemlich gleich oft und ohne $Z_{w}$ eifel unter sehr wenig verschiedenen Temperatur-Umständen beobachtet wurden, da sie demselben Sternbilde angehören, folglich in einer selben Himmelsgegend liegen. Defsungeachtet weichen sie im Fnnkeln bedeutend von einander ab, weil der eine, $\alpha$, welcher zum ersten Typus gehört, die Intensität 92 besitzt, und der andere, $\beta$, vom dritten Typus, nur die 57.

Um die Frage über den Einflufs der Temperatur voll- 
ständig zu entfernen, genügte es, die mittleren Scintillationen der drei Typen zu vergleichen mit den Brüsseler Lufttemperaturen, welche für jeden dieser Typen der Mitte desjenigen Theils eines jeden Abend entsprechen, an welchem meine Beobachtungen gemacht wurden. Da die Temperaturen bei der ersten Reihe meiner Beobachtungen, welche die zablreichste ist, in dem monatlichen Theil der Annales de l'observatoire de Bruxelles veröffentlicht sind und mir diese von dem Director Hrn. Quetelet immer gütigst mitgetheilt wurden, so berechnete ich mittelst dieser Daten die mittleren Lufttemperaturen, welche der Mitte eines jeden Beobachtungsabends entsprachen. Folgendes sind die Resultate, welche ich erhalten habe, nicht blofs in Betreff dieser, sondern auch in Betreff der mittleren Intensitäten des Funkelns der Sterne, die in der vorhergehenden Tafel aufgeführt und nach den drei Typen des P. Secchi geordnet sind. Diese Resultate beziehen sich auf die 47 Abende, welche die erste Reihe bilden.

$\begin{array}{lcccc} & \text { Erster Typus. } & \text { Zweiter Typus. } & \text { Dritter } \\ \text { Mittleres Funkeln } & \text {. } & 78 & 68 & 56 \\ \text { Mittlere Lufttemperatur } & 4^{0}, 5 & 5^{0}, 3 & 5^{0}, 8\end{array}$

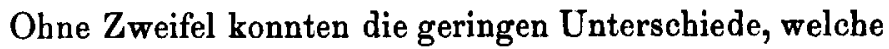
hier zwischen den Temperaturen vorkommen, nicht Ursache seyn der Verschiedenheiten, welche das Funkeln des ersten Typus gegen das des dritten auszeichnet. Fügen wir endlich hinzu, dafs die Mitteltemperaturen, die in dieser ersten Reihe den vorhin erwähnten Sternen $\alpha$ und $\beta$ Andromedae zukommen, $6^{\circ}, 7$ und $6^{\circ}, 8$ sind, obgleich die Intensitäten des Funkelns, welche diese Sterne in dieser selben Reihe charakterisiren, durch die sehr verschiedenen Werthe 82 und 59 repräsentirt werden. Die vorstehenden Thatsachen entfernen bier deutlich jede Idee des Einflussses der Lufttemperatur auf die Intensitäten des Funkelns der drei Typen.

Die vorstehenden, aus der ersten Reihe meiner Untersuchungen hervorgehenden Werthe des Funkelns sind für den zweiten und dritten Typus identisch mit den Mittel- 
werthen, welche diese in der Gesammtheit beider Reihen charakterisiren, und der dem ersten Typus angehörende Werth entfernt sich nicht bedeutend von dem Mittel 86, welches ihm durch diese Gesammtheit zukommt. Diese Uebereinstimmung trägt offenbar dazu bei, die Genauigkeit der Beobachtungen und der erhaltenen Resultate zu sichern.

Unter den Sternen des dritten Typus befinden sich sechs veränderliche. In Ermangelung entscheidender Angaben in Betreff des Einflusses, welohen die Veränderlichkeit gewisser Sterne auf die Charaktere des Funkelns baben könnte, sehe ich keinen Grund zu der Vermuthung, dafs die Phasen der Periodicität dieser sechs Sterne, die übrigens zu verschiedenen und selbst entfernten Zeiten beobachtet wurden, so mitgewirkt hätten, um ihr Funkeln und folglich den Mittelwerth der Gruppe zu schwächen. Ueberdiefs könnte dieser Einflufs der Veränderlichkeit nicht angerufen werden weder in Betreff von $\beta$ Andromedae, dessen Funkeln viel schwächer ist als das von $\alpha$ desselben Sternbildes, sondern vom ersten Typus, noch in Betreff des Arcturus und Aldebaran, Sterne von constantem Glanz und schwachem Funkeln' ).

Man hat ohne allen $\mathrm{Z}$ weifel bemerkt, dafs die Sterne $\alpha$ des Perseus und Pollux, welche vorn im zweiten Typus stehen, sich durch eben so starkes Funkeln auszeichnen wie Vega und a Andromedae, Sterne des ersten Typus. Wir können diese Eigenthümlichkeit erklären, wenn wir uns der Bemerkung des Pater Secchi erinnern, wo er sagt: „Einige wenige Spectren scheinen, statt sich deutlich einer der Kategorien anzuschliefsen, als $Z$ wischenghieder derselben $z u$ dienen*. Uebrigens bin ich weit entfernt, die uns beschäftigende Frage für gänzlich gelöst zu halten;

1) Hr. Dufour bemerkt in Betreff des Einflusses, den die Periodicität gewisser Sterne auf das Funkeln derselben haben könnte, dafs der Stern $\alpha$ oder Betelgeuze im Orion unter allen von ihm beobachteten Sternen am' unregelmälsigsten $\mathrm{za}$ funkeln scheine (Bull, de la Soc. vaudoise des sciences not. 1860 No. 47). 
ich betrachte dieses Studium nur als ersten Schritt zu einer vollständigen Lösung. Es ist unzweifelhaft, dafs man, um auf alle Einzelheiten der uns beschäftigenden Aufgabe zu antworten, nothwendig Rücksicht zu nehmen babe auf die Anzahl und Breite der Linien in den Spectren der verschiedenen Sterne jedes Typus und besonders auf die mehr oder weniger Dunkelheit dieser Linien. Diese Lichtschwächung ist eine zarte Frage der Spectral-Analyse, die man hier sehr berücksichtigen muls, denn, wenn es in den Sternspectren Linien giebt, die nicht absolut dunkel sind, so giebt es auch nothwendig cinige Lichtstrablen in den Lücken, welche diesen Linien in den durch unsere Atmosphäre gehenden Farbenbündeln der Sterne entsprechen. In diesem Falle werden die Häufigkeit und die Charaktere des Funkelns dieser Sterne offenbar afficirt seyn durch das Daseyn einiger Lichtstrahlen in diesen Lüoken. Bemerken wir übrigens, da/s das Daseyn von Strahlen in veränderlicher Menge unzweifelhaft ist in den Lücken, die den mehr oder weniger dunklen Nebulositäten in den Spectren der Sterne des dritten Typus entsprechen. Fugen wir endlich hinzu, dafs diese selben Strablen, vermöge ihrer mehr oder weniger hervortretenden Gegenwart auch mitwirken zu dem End-Eindruck, der, bei jeder Farbenveränderung, aus der Mischung der verschiedenen Farben auf der Netzhaut entspringt.

Nachdem wir im Vorstehenden die Betrachtungen vereinigt haben, welche mit Sicherheit zu beweisen streben, dafs zwisohen der Häufigkeit der Farbenveränderungen beim Funkeln der Sterne und der spectral-analytischen Beschaffenbeit ihres Lichts ein inniger Zusammenhang existirt; nachdem wir gewisse Einflüsse, die bei diesem $\mathrm{Phä-}$ nomèn mitwirken, auf ihren wahren Werth zurückgeführt haben, mufs ich bemerken, dafs diese Abhängigkeit zwischen den beiden $\mathrm{Phänomenen} \mathrm{im} \mathrm{vollen} \mathrm{Einklang} \mathrm{mit} \mathrm{Hrn.}$ Dufour's erstem Gesetz steht, welches sagt, dafs die rothen Sterne weniger funkeln als die gelben. In der 
That, die Sterne des dritten Typus, die nach P. Secchi roth oder orange sind, sind auch genau diejenigen, die nach meinen Versuchen am wenigsten funkeln. Diese errichten äberdiefs einen ähnlichen Unterschied zwischen den rothen und den gelben Sternen oder des zweiten Typus, was auch die Thatsache eines Unterschieds, den ich in Betreff des Funkelns der gelben Sterne relativ zu den ersteren vermuthet hatte, bestätigt. ${ }^{1}$ )

Nicht blofs bestätigen meine Untersuchungen das erste Gesetz des Hrn. D ufour und dehnen es auf andere Sterne aus, sondern sie geben auch eine vollständige Erklärung desselben. Es ist wichtig hier zu sagen, dafs Hr. Dufour, welcher die A rago' sche Erklärung des Funkelns annimmt, indem er dasselbe als eine Folge des Interferenzprincips betrachtet, schon die Thatsache, dafs die rothen Sterne weniger funkeln als die weifsen, durch die Bemerkung erklärt, dafs, da die rothe Welle die grölste unter den Lichtwellen ist, zur Interferenz der rothen Strahlen beträchtlichere Ablenkungen, gröfsere Störungen in der Atmosphäre erforderlich seyen. Da ich diese Erklärung nicht annehmen kann, so habe ich in der erwähnten Arbeit das häufigere Funkeln der weifsen Sterne davon abgeleitet, dafs, bei gleicher Entfernung vom Beobachter, die totale Dirergenz der von einem weilsen Stern ausgehenden und von der Atmosphäre zerstreuten Farbenbündeln viel gröfser ist als wenn der Stern roth ist, oder seine Bündel, unter sonst gleichen Umständen, aus einer grörsern Menge von rothen Strahlen bestehen. In Folge dieses Unterschiedes sind die von einem weifsen Stern ausgehenden Strahlen, da sie die zahlreichsten und folglich am meisten durch Dispersion ausgebreiteten sind, auch häufig der Auffangung durch den Durchgang von Luftwellen ausgesetzt. Diese Erklärung, welche hier ihre ganze Wichtigkeit und ihren reellen Werth behält, wird vervollständigt durch die Thatsache des Einflusses der in den Sternstrahlen vorhandenen

1) Notice sur la scintillation des étoiles (Bull. de l'acad. roy. de Belgique, Ser. II, T. XXV, p. 635). 
Lücken, welche mehr oder weniger breit und zahlreich sind, je nach der Dicke und Menge der Spectrallinien des Sterns.

Was das dritte Gesetz des Hrn. Du four betrifft, welches er so ausdrückt: „Aufser den Farben giebt es noch zwischen dem Funkeln der Sterne wesentliche Unterschiede, die von den Sternen selbst herzurühren scheinen" so lehrt uns die Spectral-Analyse diese Unterschiede kennen; wie aus allem Vorstehenden hervorgeht ${ }^{1}$ ).

Zum Schlusse dieses Studiums will ich noch die Aufmerksamkeit der Personen, die sich dafür interessiren, auf die Wichtigkeit der mit blofsem Auge gemachten Beobachtungen des Funkelns lenken, deren Hr. Dufour 13000 gesammelt hat, will die Uebereinstimmung hervorheben, die zwischen den relativen Intensitäten, die er in den Annales de la société vaudoise, aus den mit blofsem Auge gemachten Beobachtungen an sechs Sternen hergeleitet hat, und den absoluten Werthen besteht, die ich den mittleren Intensitäten des Funkelns derselben Sterne beilege.

In der folgenden Tafel sind diese Resultate neben einander gestellt, zugleich mit dem Typus, zu welchem jeder Stern nach P. Secchi gehört.

\begin{tabular}{|c|c|c|c|}
\hline Sterne & $\begin{array}{c}\text { Relative Intensität } \\
\text { des Funkelns } \\
\text { mit blofsem } \\
\text { Auge beobachtet } \\
\text { (Dufour) }\end{array}$ & $\begin{array}{c}\text { Farbenverände- } \\
\text { rungen beobachtet } \\
\text { mit dem Scintillo- } \\
\text { meter } \\
\text { (Montigny) }\end{array}$ & $\begin{array}{l}\text { Typus } \\
\text { zu dem der } \\
\text { Stern } \\
\text { gehört. }\end{array}$ \\
\hline Prokyon & 113 & 103 & Erster \\
\hline Vega & 110 & 98 & Erster \\
\hline Capella & 101 & 77 & Zweiter \\
\hline Aldebaran & 99 & 59 & Dritter \\
\hline Arcturus & 81 & 59 & Dritter \\
\hline$\alpha$ Orion (Betelgeuze) & 90 (appr) & 64 & Dritter \\
\hline
\end{tabular}


höhen, um so z. B. das Funkeln des Prokyon auf die Dufour'sche Zahl 113 zu erheben, so erhalten wir respective die Werthe $113,108,85,65$ und 70. Diese Umformung zeigt uns, dafs das Funkeln von Prokyon und Vega nach beiden Methoden genau in demselben Verbältnifs steht; und dals, wenn das Funkeln der übrigen Sterne nicht genau in vollkommen gleichen Verhältnissen steht, es wenigstens derselben Ordnung folgt, Betelgeaze mitbegriffen, dessen Intensität und Veränderungen hervorstehender sind, wie, nach beiden Reihen, die von Arcturus. Diese Uebereinstimmungen bieten in Bezug auf die beiden angewandten Methoden um so mehr Interesse, als die Beobachtungen unter so verschiedenen Klimaten, wie das der Schweiz und das von Belgien, gemacht worden sind.

\section{Ueber Nadel_Inklinatorien;}

con Karl Braun, S. J. in Karlsburg bei When.

Im vorhergehenden Band dieser Annalen habe ich in dem Aufsatz „Studien aber erdmagnetische Messungen“ die aufserordentliche Genauigkeit der neueren englischen Inklinatorien von $D$ over ziemlich eingehend besprochen. Ich fürchte, dafs die Resultate meiner Untersuchungen eben wegen ihrer Aufsergewöhnlichkeit vielleicht nicht unzweifelhaften Glauben finden dürften. Deshalb gebe ich hier in Kurzem eine Bestätigung derselben.

Die Central-Anstalt für Meteorologie und Erdmagnetismus in Wien besitzt seit 1870 ebenfalls ein Instrument von Dover, und die damit ausgeführten Messungen liegen in zwei Bänden der Jahrbücher derselben Anstalt veröffentlicht vor.

Eine oberflächliche Betrachtung der Zahlen in Bd. VII reicht hin, den ganz aufserordentlichen Unterschied ins 